Check for updates

Cite this: RSC Adv., 2019, 9, 25576

\title{
Full-color-emitting (CulnS 2 )ZnS-alloyed core/shell quantum dots with trimethoxysilyl end-capped ligands soluble in an ionic liquid
}

\author{
Huiqing Wang, (DD *ab Jiayuan Hu, ${ }^{a}$ Min Zhu, ${ }^{a}$ Yucheng Li, ${ }^{a}$ Hao Qian, ${ }^{a}$ Xiaofei Shen, ${ }^{a}$ \\ Falk Liebner (D) ${ }^{* b}$ and Thomas Rosenau ${ }^{\mathrm{b}}$
}

Zinc-copper-indium sulfide (ZCIS)-alloyed quantum dots are emerging as a new family of low toxic I-III-VI semiconductors due to their broad and color-tunable emissions as well as large Stokes shifts. Here, we fabricated a series of ZCIS QDs with tunable PL wavelengths and band-gap energies via a facile strategy by varying the ratio of $\mathrm{A} 1-3$ stock $\left(\mathrm{Cu}^{+} / \mathrm{In}^{3+}\right)$ to the $\mathrm{B}$ stock $\left(\mathrm{Zn}^{2+}\right)$ content. The $\mathrm{ZnS}$ shell was formed to improve the PL emission efficiency of the core nanoparticles and the PL emission wavelength of the resulting ZCIS/ZnS NCs gradually blue-shifted with an increase in the number of shell layers, resulting in a wide range of emissions from $800 \mathrm{~nm}$ to $518 \mathrm{~nm}$ that can be tuned by the core compositions or shell layer numbers for ZCIS/ZnS. Finally, the long-chain ligands dodecanethiol/octadecylamine on the quantum dots' surface were efficiently replaced by (3-mercaptopropyl)trimethoxysilane, thus enabling their solubility in an ionic liquid, which was confirmed via GC-MS. It also benefited for the co-dissolution of the polymers and chemical binding with other materials through the reactive silanol group, which provide stable and well-distributed ZCIS/ZnS QDs composites or surface coating by the QDs.

Received 24th April 2019

Accepted 24th July 2019

DOI: $10.1039 / c 9 r a 03066 b$

rsc.li/rsc-advances
$\left(E_{\mathrm{g}}=3.7 \mathrm{eV}\right)$ is of special interest for very low $(\sim 2.2 \%)$ lattice mismatch due to their similar crystallographic structures and low toxicity. The band gap of $\mathrm{CuInS}_{2}-\mathrm{ZnS}$ alloys (ZCIS) can vary between 1.55 and $3.7 \mathrm{eV}$. Fabricating ZCIS is promising for applications in biolabelling, photocatalysis, solar energy conversion, display technology, and light-emitting diodes. Several literature reports on the direct synthesis of ZCIS NPs have been described..$^{15-20}$ Typically, the thermolysis of complexes of dithiocarbamate with $\mathrm{Zn}^{2+}, \mathrm{Cu}^{2+}$, and $\mathrm{In}^{3+}$ in the presence of oleylamine as an activating agent formed homogeneous nanocrystals. ${ }^{19}$ Another approach is a two-step procedure, in which the $\mathrm{CuInS}_{2}$ crystallites are synthesized first and the alloy particles are obtained by cation exchange. ${ }^{15,21}$

Herein, a facile strategy was reported for the synthesis of the ZCIS-alloyed core nanoparticles by varying the ratio of the stock $\mathrm{A}$ $\left(\mathrm{Cu}^{+} \mathrm{In}^{3+}\right)$ to stock B $\left(\mathrm{Zn}^{2+}\right)$ to rapidly synthesize a series of ZCIS with different compositions using 1-dodecanethiol as the sulfur source and as the ligand. The overcoating of fluorescent mc-NCs with semiconductors of higher band-gap energy is a common way to minimize the non-radiative transitions caused by surface defect trapping to enhance the PL intensity. ${ }^{22}$ Here, batch feeding approach was followed for the core growth to deposit a ZnS shell around the ZCIS core NCs with designed layers, which also induced a blue shift as reported and was beneficial in widening the PL range of the ZCIS/ZnS core/shell QDs to provide tunable photoluminescence (PL) emission property.

The ligand covered on the QD surface limited the suitability for further applications. ${ }^{23}$ Thus, an effective ligand exchange 
was needed to transfer the QDs to the target solvent for their application. The ZCIS QDs obtained by colloidal synthesis at high temperature are usually covered by long-chain ligands, for example, the ligands replaced by mercaptohexanol ${ }^{24}$ enabled the solubility in methanol, ethanol, and dimethylformamide, and the ligands exchanged with lipoproic acid, ${ }^{25}$ poly(maleic anhydride-alt-1-octadecene), ${ }^{26}$ or dihydrolipoic acid ${ }^{27}$ render the particles water-soluble. ${ }^{28,29}$

Herein, we successfully exchanged the ligand 1-dodecanethiol by 3-mercaptopropyl-trimethoxysilane with the help of the ionic liquid 1-hexyl-3-methylimidazolium chloride as the new solvent. The 3-mercaptopropyl-trimethoxysilane (MPTMS) contains bi-functional groups that can bind to glass surfaces or metal oxide films and polymers via hydrogen bonds and covalent bonds. 3-Mercaptopropyltrimethoxysilane had been applied to encapsulate gold nanoparticles, ${ }^{30}$ sliver nanoparticles, ${ }^{31}$ and so on. Herein, for the first time, it has been applied to encapsulate ZCIS QDs. Further, here the ligand exchange reaction with 3-mercaptopropyl-trimethoxysilane also provide the QDs solubility in the ionic liquid, which is called as a super solvent for many materials and natural polymers such as cellulose,${ }^{32-34}$ collagen ${ }^{35}$ keratin, ${ }^{36}$ and chitin, ${ }^{37}$ which are not soluble in common organic solvents but can be dissolved in ionic liquid; some synthetic polymers are also soluble in the ionic liquid. ${ }^{38}$ Therefore, here the MPS-ZCIS/ZnSQDs were applied in homogeneous QD polymer composites or stable surface coating by QDs on other matrices.

\section{Experimental section}

\section{Chemicals}

Copper(I) iodide (CuI, 99.99\%), indium(III) acetate (In(OAc) 99.99\%), zinc(II) acetate ( $\left.\mathrm{Zn}(\mathrm{OAc})_{2}, 99.99 \%\right)$, 1-dodecanethiol (99.9\%), 1-octadecylamine (97\%), 1-octadecene (90\%), and $\gamma$ (mercaptopropyl)trimethoxysilane (95\%) were purchased from Sigma-Aldrich Corp. (USA) and used without further purification.

Stock solution series A1-3. The starting materials included the following: (0.05 $\mathrm{M} \mathrm{Cu}^{+} ; \mathrm{Cu}:$ In ratios of $1: 1,1: 2$, and $\left.1: 4\right)$; $0.095 \mathrm{~g}(0.5 \mathrm{mmol})$ of CuI, $0.292 \mathrm{~g}(1 \mathrm{mmol})$ of $\mathrm{In}(\mathrm{OAc})_{3}, 2.5 \mathrm{~mL}$ of 1-mercapto- $n$-dodecane, and $7.5 \mathrm{~mL}$ of 1 -octadecene. These were placed in a pre-dried three-necked round-bottom flask and degassed under vacuum at $80{ }^{\circ} \mathrm{C}$ for $60 \mathrm{~min}$. The mixture was then heated to $160{ }^{\circ} \mathrm{C}$ in an argon atmosphere and held at this temperature for about $30 \mathrm{~min}$ until a clear green solution was formed. The resulting CuIn stock solution A2 (0.05 M, Cu/In ratio of $1: 2$ ) was stored at $50{ }^{\circ} \mathrm{C}$ under the argon atmosphere until further processing.

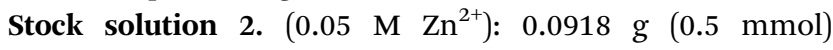
$\mathrm{Zn}(\mathrm{OAc})_{2}, 0.5799 \mathrm{~g}$ (2 mmol) 1-octadecylamine, and $9.8 \mathrm{~mL} \mathrm{1-}$ octadecene were treated as described above to obtain a colorless solution.

\section{Preparation of ZnCuInS (ZCIS) core nanoparticles}

In a typical procedure, $1.0 \mathrm{~mL}$ of the stock solution $\mathrm{A} 1$ $\left(0.05 \mathrm{mmol} \mathrm{Cu}{ }^{+} \mathrm{I}^{3+}\right), 0.7 \mathrm{~mL}$ of the stock solution $\mathrm{B}(0.035 \mathrm{mmol}$
$\mathrm{Zn}^{2+}$ ), and $1 \mathrm{~mL}$ dodecanethiol were mixed in $8.0 \mathrm{~mL}$ of ODE in a $50 \mathrm{~mL}$ three-necked round bottom flask. This was subjected to three cycles of degassing and purging with argon on a Schlenk line, and then heated to $230{ }^{\circ} \mathrm{C}$ in an argon atmosphere under vigorous stirring. The aliquots of each batch were taken at different time intervals and injected into cold toluene to terminate the growth of NCs and to record their optical spectra. When the ideal fluorescent properties were achieved, the mixture was cooled in an ice water bath with stirring and $10 \mathrm{~mL}$ of toluene was added to dilute it thereafter. The resulting ZCIS NCs $(2 \mathrm{~mL})$ were pipetted into $15 \mathrm{~mL}$ centrifuge tubes, precipitated with $8 \mathrm{~mL}$ acetone, and then isolated by centrifugation and decantation. The mc-NCs were further purified by repeatedly dispersing the material in toluene and precipitating it in ethanol and acetone. The purified NCs were redispersed in toluene for the optical and morphological measurements or dried in vacuum for XRD measurements.

A series of ZCIS core nanoparticles were prepared with the stock solutions A1-3 and B with $\mathrm{Zn}^{2+}: \mathrm{Cu}^{+}$in the mole ratios of $1,0.7,0.5,0.25$, and 0 at the reaction temperatures of $210{ }^{\circ} \mathrm{C}$, $230{ }^{\circ} \mathrm{C}$, and $250{ }^{\circ} \mathrm{C}$ with $0.25-3 \mathrm{~mL}$ of DDT.

ZCIS/CIS "core"-ZnS "shell" nanoparticles. The CIS or ZCIS core particles were grown at $230{ }^{\circ} \mathrm{C}$ for $60 \mathrm{~min}$ as described above. After raising the temperature to $240{ }^{\circ} \mathrm{C}$, the deposition of $\mathrm{ZnS}$ on the surface of respective core templates was accomplished by the portion-wise addition of $0.4 \mathrm{~mL} \mathrm{Zn}$ stock solution, which was injected into the reaction mixture in 5 batches at intervals of $30 \mathrm{~min}$ each. The stock A CIS solution can still contain enough sulfur precursor, so the addition of sulfur is not always necessary. ${ }^{39}$

To monitor the reaction, the aliquots were taken before injecting a new batch of $\mathrm{Zn}$ stock and their corresponding optical spectra were recorded accordingly. The purification method of ZCIS/ZnS NCs was similar to that of ZCIS NCs.

Solubilization of ZCIS/ZnS nanoparticles in ionic liquids by ligand exchange with $\gamma$-(mercaptopropyl)trimethoxysilane. A solution of $0.05 \mathrm{~mL} \gamma$-(mercaptopropyl)trimethoxysilane in $5.0 \mathrm{~mL}$ 1-hexyl-3-methylimidazolium chloride was added to a $5.0 \mathrm{~mL}$ solution of ZCIS/ZnS core/shell nanoparticles solubilized in toluene by the lipophilic 1-dodecanethiol ligands. The resulting two-phase system was vigorously stirred at ambient temperature for $30 \mathrm{~min}$, whereupon the mc-NCs moved from the supernatant toluene into the lower ionic liquid phase. The supernatant toluene was separated out for GC-MS detection to confirm the ligand exchange and the ionic liquid phase containing the mc-NCs was collected and stored in cool and dry conditions.

\section{Characterization}

For optical analysis, the as-prepared solutions were diluted with toluene to an optical density of approximately $0.1-0.2$. The absorption spectra of the ZCIS-alloyed core and ZCIS/ZnS core/ shell QDs were collected via UV-visible absorption spectroscopy (Lambda 35, PerkinElmer spectrometer). PL emission spectra were recorded using a fluorescence spectrometer (LS55, PerkinElmer, USA). The PL QYs of the QDs dispersed in chloroform 
were measured by comparing their integrated emissions with those of a rhodamine 6G (QY of 96\%) ethanol standard with an identical optical density of 0.05. X-ray diffraction (XRD, PW1710, Philips Co.) and transmission electron microscopy (TEM) (JEOL JEM-1400) were used for the morphological and structural analyses. For TEM, the NCs were deposited from the dilute toluene solutions onto copper grids with carbon supports by slowly evaporating the solvent in air at room temperature, followed by a second carbon film coating. AFM experiments were performed using a Nanoscope III AFM (Digital Instruments, Santa Barbara, CA) fitted with a $15 \times 15 \mu \mathrm{m}$ scanner. Silicon nitride tips (Digital Instruments) with a spring constant of $0.06 \mathrm{~N} \mathrm{~m}^{-1}$ and a nominal radius of 5-40 nm were used; the images were recorded in both the height and amplitude modes.

The ligand exchange behavior was analyzed by GC-MS (Agilent 5975B inert Series GC/MS System) using $500 \mu \mathrm{g}$ Br-decane in $250 \mu \mathrm{L}$ toluene solution as the internal standard. The original ligand 1-dodecanethiol was dispersed again from the QDS into the toluene after the ligand exchange and different concentrations of the decanted toluene were collected for measurement; the amount of ligand exchange was detected by GC-MS as an indirect confirmation of the ligand exchange behavior.

\section{Results and discussion}

The ZCIS NCs were prepared in 1-octadecene using In and Zn acetates and copper iodide as the cationic precursors in the presence of 1-amino- $n$-octadecane and 1-dodecanethiol without sulfur injection; 1-dodecanethiol served as the sulfur source. This simultaneously stabilized the resulting NCs. ${ }^{40}$ The PL properties (PL peak position and PL intensity) of the resulting ZCIS NCs were strongly dependent on the experimental variables, such as $\mathrm{Zn}$ content, $\mathrm{Cu}$ : In ratio, 1-dodecanethiol concentration, and reaction temperature. Since the PL QYs of all the as-prepared ZCIS NCs under different experimental conditions were not high (usually less than 5\%) and could not approach the desired value for practical applications, the PL QYs were not considered as an experimental variable. The PL QYs were mainly improved via the construction of $\mathrm{ZnS}$ shells around the ZCIS core nanoparticles, as discussed below. Hereafter, we only describe the dependence of PL wavelength on the experimental variables.

\section{Effect of Zn content}

We attempted to tune the PL wavelength of the resulting ZCIS NCs by controlling the $\mathrm{Zn}: \mathrm{Cu}:$ In ratio while keeping the other experimental variables constant. Different $\mathrm{Zn}: \mathrm{Cu}:$ In ratios were achieved by controlling the concentration of the precursors during synthesis. Herein, the molar ratio of $\mathrm{Zn}^{2+}$ to $\mathrm{Cu}^{+}$in the precursor solution was varied from $0: 1$ to $1: 1$, and equimolar concentrations of $\mathrm{Cu}^{+}$and $\mathrm{In}^{3+}$ were used in each case. The color of the quantum dot suspensions in toluene changed from light to dark, as observed in daylight with the increase in reaction time, as shown in Fig. 1A.
Fig. 1A shows that for a reaction time of $90 \mathrm{~min}$, the color of the QD suspension in toluene changes from brownish $\left(\lambda_{\mathrm{em}}=\right.$ $685 \mathrm{~nm} ; \mathrm{Cu}: \mathrm{In}: \mathrm{Zn}=1: 1: 0)$ to reddish $\left(\lambda_{\mathrm{em}}=628 \mathrm{~nm}\right.$; $\mathrm{Cu}: \mathrm{In}: \mathrm{Zn}=1: 1: 0.2)$, orange $\left(\lambda_{\mathrm{em}}=596 \mathrm{~nm} ; \mathrm{Cu}: \mathrm{In}: \mathrm{Zn}=\right.$ $1: 1: 0.5)$, yellow $\left(\lambda_{\mathrm{em}}=575 \mathrm{~nm} ; \mathrm{Cu}: \mathrm{In}: \mathrm{Zn}=1: 1: 0.7\right)$, and faint yellow $\left(\lambda_{\mathrm{em}}=555 \mathrm{~nm}\right.$; Cu $\left.: \mathrm{In}: \mathrm{Zn}=1: 1: 1\right)$ with the increasing amount of $\mathrm{Zn}$ in the composition. The evident blueshift in the PL emission wavelengths of the NCs results from an increase in the band-gap energies with the increase in the $\mathrm{Zn}$ content. This is ascribed to the incorporation of ZnS into CIS. The band gap of bulk $\mathrm{ZnS}(3.6 \mathrm{eV})$ is markedly higher than that of $\mathrm{CuInS}_{2}(1.5 \mathrm{eV})$, and $\mathrm{Zn}^{2+}$ statistically replaces $\mathrm{Cu}^{+}$and $\mathrm{In}^{3+}$. Thus, it is reasonable to assume that the band gap of the $\mathrm{ZnSCuInS}_{2}$ alloy is higher than that of CIS. This strategy for band gap tuning has been commonly used in ZnS-CdS and ZnSe-CdSe-alloyed nanostructures. ${ }^{41}$

The fluorescence emitting wavelength of the ZCIS cores were detected at $\lambda_{\text {ex }}=470 \mathrm{~nm}$, as shown in Fig. 1B. It was observed that it is difficult to make Zn-free CIS NCs with a particle size that afforded an emission wavelength below $600 \mathrm{~nm}$ due to rapid particle growth. On the other hand, the addition of $\mathrm{Zn}$ in increasing amounts reduced the growth rate, as seen in the decreasing slope of the curves. The slower growth rate was beneficial for controlling the emission wavelength of the quantum dots. Furthermore, the addition of $\mathrm{Zn}$ facilitates the formation of QDs with a wider emission wavelength range. By changing the QD composition from $\mathrm{Cu}: \mathrm{In}: \mathrm{Zn}=1: 1: 0.2$ to $\mathrm{Cu}: \mathrm{In}: \mathrm{Zn}=1: 1: 1$, the minimum emission wavelength could be lowered from 580 to $530 \mathrm{~nm}$.
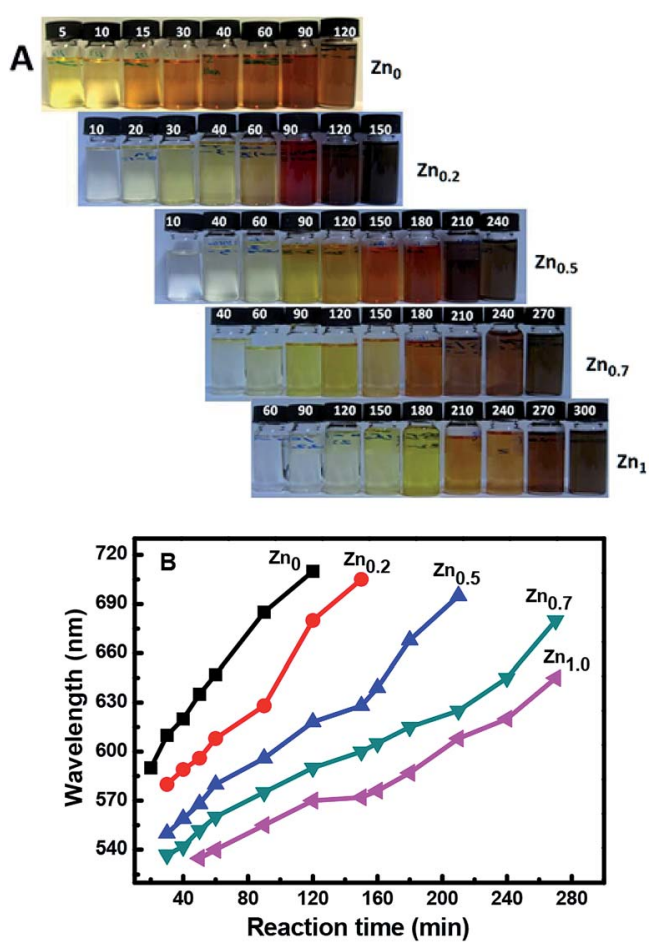

Fig. 1 Visible light photographs (A) and emission wavelengths (B) of the ZCIS core nanoparticles with different $\mathrm{Zn}$ ratios with increase in time at $230{ }^{\circ} \mathrm{C}$. 


\section{Effect of the $\mathrm{Cu}$ : In ratio}

Fig. 2 shows that the particle growth rate not only depends on the amount of added $\mathrm{Zn}$ but also on the $\mathrm{Cu} / \mathrm{In}$ ratio. The QD growth rate at $30 \mathrm{~min}$ of reaction time is significantly higher at a $\mathrm{Cu}:$ In ratio of $1: 4$ compared to that at the $\mathrm{Cu}:$ In ratio of $1: 1$. This difference increased with increasing amounts of $\mathrm{Zn}$. At a fixed reaction time/temperature and at a $\mathrm{Zn}$ content of 0.7 , the fluorescent peak position at $\mathrm{Cu} / \mathrm{In}=1: 1$ is $537 \mathrm{~nm}$, while at $\mathrm{Cu} / \mathrm{In}=1: 2$ and $\mathrm{Cu} / \mathrm{In}=1: 4$ it is $574 \mathrm{~nm}$ and $599 \mathrm{~nm}$, respectively. This indicates that at a higher In content the nanoparticles grew faster and showed higher emission wavelengths. Thus, by adjusting the $\mathrm{Zn}$ content, the difference in the peak positions lessened more for the $\mathrm{Cu} / \mathrm{In}=1: 4$ samples than for the $\mathrm{Cu} / \mathrm{In}=1: 1$ samples. Therefore, adjusting the $\mathrm{Zn}$ content could produce QDs with an emission wavelength ranging from $585 \mathrm{~nm}$ to $635 \mathrm{~nm}(\mathrm{Cu} / \mathrm{In}=1: 4$ with), $554 \mathrm{~nm}$ to $625 \mathrm{~nm}(\mathrm{Cu} / \mathrm{In}=1: 2)$, and $537 \mathrm{~nm}$ (or less) to $605 \mathrm{~nm}(\mathrm{Cu} / \mathrm{In}=$ $1: 1)$. However, at the same reaction time, a higher In content could significantly improve the quantum yield (Fig. 2B); the emission intensity at $\mathrm{Cu} / \mathrm{In}=1: 4$ is much higher than that at a lower In content. These In-rich effects have also been reported for the CuInS system. ${ }^{42}$ However, the QY at $\mathrm{Cu} / \mathrm{In}=1: 1$ can be improved by the subsequent deposition of the $\mathrm{ZnS}$ shell.

\section{Effect of 1-dodecanethiol concentration}

Fig. 3A shows that when the other reaction variables were held constant, such as the $\mathrm{Zn} / \mathrm{Cu} / \mathrm{In}$ ratio, the $\mathrm{PL}$ peak position of the resulting ZCIS NCs strongly depended on the thiol concentration with the increase in the reaction time. The dependence could be divided into two behaviors: when the amount of thiol was less than $1 \mathrm{~mL}$, it barely had any effect on the PL peak position; however, when the amount of thiol was more than 1
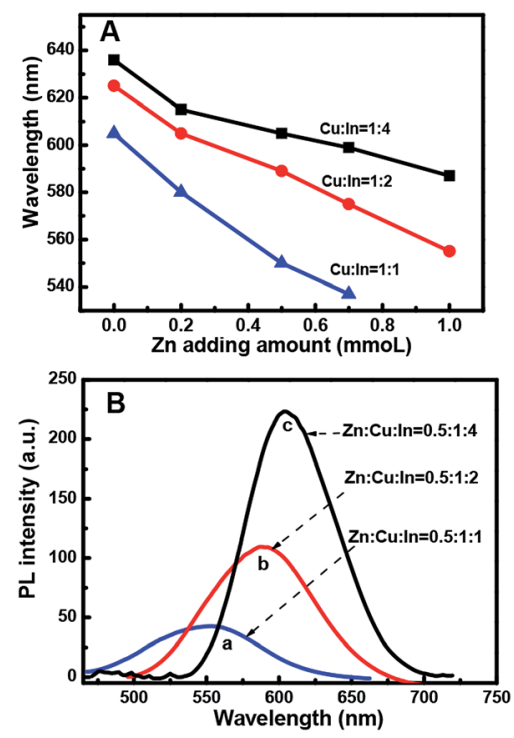

Fig. 2 (A) Emission wavelength of the ZnCulnS alloy nanoparticles with different zinc quantities and various $\mathrm{Cu} / \mathrm{In}$ ratios at $230^{\circ} \mathrm{C}$, grown for 30 min with $0.5 \mathrm{~mL}$ 1-dodecanethiol. (B) PL emission spectra of the ZnCulnS alloy nanoparticles with various $\mathrm{Cu} / \mathrm{ln}$ ratios having $\mathrm{Zn}$ content of 0.5 . All were grown for $30 \mathrm{~min}$ at $230{ }^{\circ} \mathrm{C}$.
$\mathrm{mL}$, the PL peak shifted significantly towards shorter wavelengths. However, the concentration effect of $3 \mathrm{~mL}$ thiol is almost the same as that of $2 \mathrm{~mL}$ thiol. This could be ascribed to the composition variation in the presence of thiol contents over $1 \mathrm{~mL}$.

\section{Effect of reaction temperatures}

Fig. 3B shows the dependence of PL wavelength on temperature during QD preparation. The shift of the PL peak towards longer wavelengths suggests an exponential increase in the growth rate of the QDs at reaction temperatures above $210^{\circ} \mathrm{C}$. Therefore, it becomes increasingly difficult to control the growth of QDs at high temperatures and the NCs precipitated within the reaction mixture at $250{ }^{\circ} \mathrm{C}$ in $1 \mathrm{~h}$. Since 1-dodecanethiol acts both as a sulfur precursor and a stabilizing ligand during the reaction, its gradual decomposition at elevated temperatures destabilizes the colloids. However, due to the much slower growth rate and time consuming nature of QD formation with a weak PL emission at $210{ }^{\circ} \mathrm{C}$, the reaction was better at $230{ }^{\circ} \mathrm{C}$, where the growth was sufficiently controllable and yielded a comparably stronger PL emission band.

\section{ZCIS/ZnS core/shell structure}

Significant increase in the fluorescence emission intensity. Coating the ZCIS particles with ZnS shells showed a beneficial effect on the photoluminescence properties of the cores; more the shell layers, higher the emissions of the ZCIS/ZnS, as shown in Fig. 4A when the number of shell layers grows from one (a) to six (f). On the other hand, a blue-shift of the emission (Fig. 4A) and the absorption (Fig. 4B) band can be observed. Usually, most type I core-shell structures showed a red-shift of the
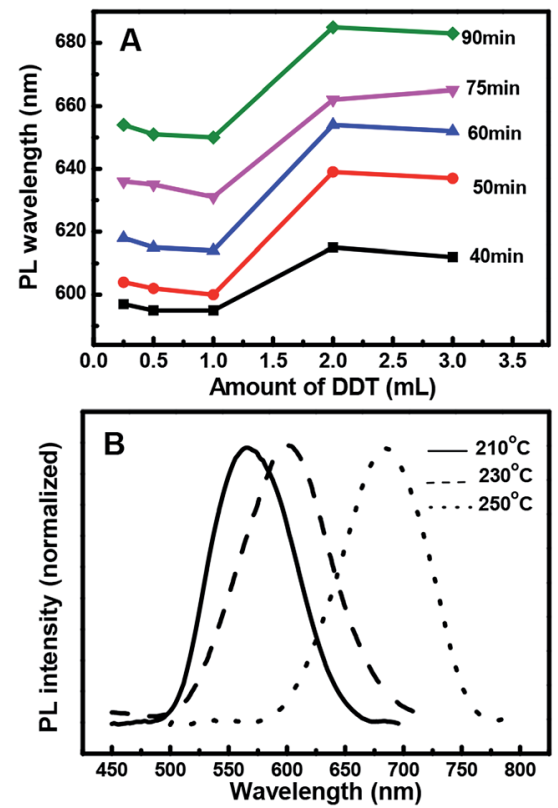

Fig. 3 (A) PL emission wavelength throughout the reaction process of the $\mathrm{Zn}$-alloyed $\mathrm{CulnS}_{2}$ nanoparticles with $\mathrm{Zn} / \mathrm{Cu} / \mathrm{In}=0.2: 1: 2$ with different 1-dodecanethiol amounts. (B) Effect of reaction temperature during QD preparation on their photoluminescence wavelength. 

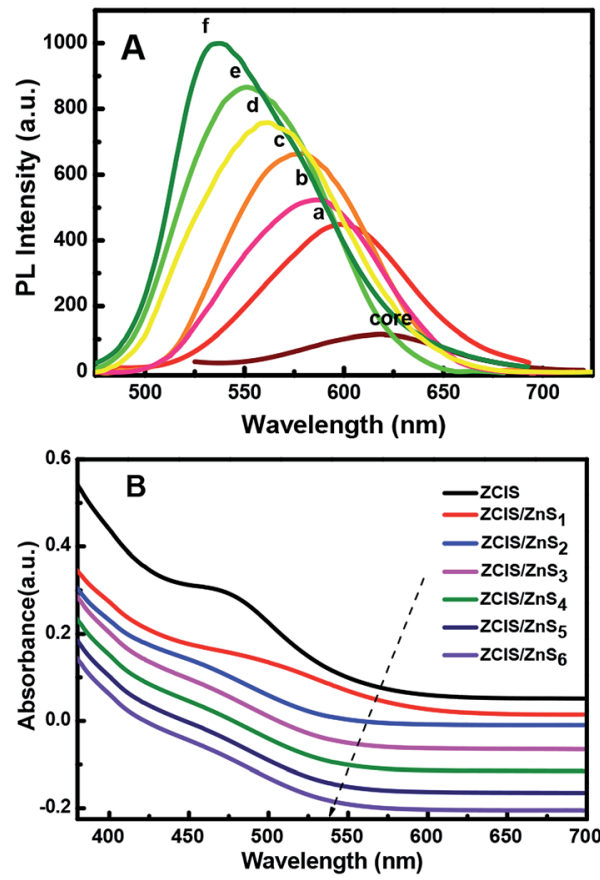

Fig. $4 \mathrm{PL}$ emission spectra (A) of the resulting alloyed NCs with increasing number of ZnS shells deposited around the ZCIS core from one (a) to six (f) and their UV absorption (B).

absorption and emission bands after shell coating due to a slight delocalization of the charge carriers into the shell layer. ${ }^{43}$ Such a blue-shift was reported in some other CIS-ZnS core-shell QDs cases. ${ }^{44}$ Several different explanations for the blue-shift have been suggested, including surface reconstruction, ${ }^{45}$ inter-diffusion of $\mathrm{Zn}$ atoms,${ }^{46}$ or etching of the core material. ${ }^{47}$ The absorption spectra seen in Fig. $4 \mathrm{~B}$ eliminate the possibility of $\mathrm{Zn}$ diffusion-associated blue-shift because the corresponding change in the band gap of the core/shell QDs was not observed. Surface reconstruction might be a cause of these findings, but it is difficult to claim without an appropriate theoretical or experimental understanding. Recently, it was found that cation exchange in the outer layer of the CIS nanocrystals explained the large blue-shift observed for the CIS-ZnS core-shell structures. ${ }^{48}$

Thus, the emission wavelength and QY of the resulting ZCIS/ $\mathrm{ZnS}$ core/shell structure can be tuned by varying the band gap of the starting ZCIS core nanoparticles and via the batch-wise addition of $\mathrm{ZnS}$ for shell growth. Fig. 5A and B show that the emission wavelength of the ZCIS/ZnS core/shell NCs prepared from ZCIS core NCs with different $\mathrm{Zn} / \mathrm{Cu}$ ratios have remarkable fluorescent emission brightness and cover most of the visible light region (from $527 \mathrm{~nm}$ to $700 \mathrm{~nm}$ ).

Fig. 6 shows the wide-field TEM images of the $\mathrm{ZCIS}_{620}$ core nanoparticles prepared from $\mathrm{Zn} / \mathrm{Cu} / \mathrm{In}=0.2: 1: 2$ at $230{ }^{\circ} \mathrm{C}$ in $1 \mathrm{~h}$ with an average size of $4 \mathrm{~nm}$ (Fig. 6A) as well as the corresponding $\mathrm{ZCIS} / \mathrm{ZnS}_{575}$ core/shell nanoparticles derived from the initial $\mathrm{ZCIS}_{620}$ cores via the three-step growth of the ZnS shell at $230{ }^{\circ} \mathrm{C}$ with an average size of $10 \mathrm{~nm}$ (Fig. 6D). The AFM measurements also confirmed the change in size during the shell growth, as shown in Fig. 6B and C. The diameter of the
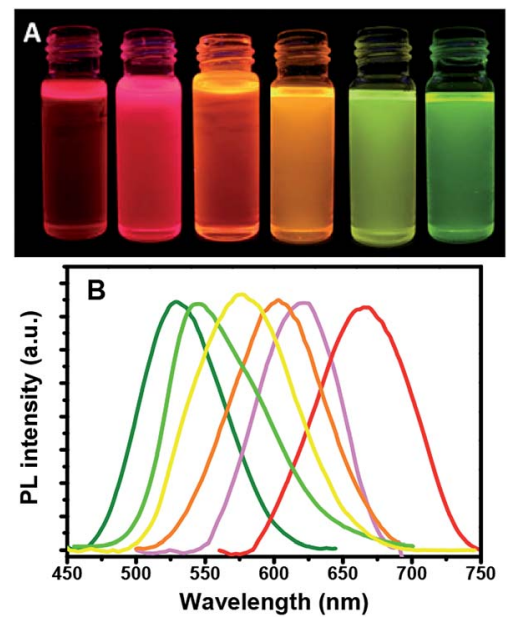

Fig. 5 Normalized PL spectra $\left(\lambda_{\text {ex }}=365 \mathrm{~nm}\right.$ ) of ZCIS/ZnS core/shell $\mathrm{NCs}$ obtained from ZCIS core with different $\mathrm{Zn} / \mathrm{Cu}$ ratios (A) and the representative emission colors of $\mathrm{NC}$ dispersion in toluene under the radiation of a UV lamp (B).

$\mathrm{ZCIS}_{620}$ core particles is about $3 \pm 0.4 \mathrm{~nm}$, while the corresponding $\mathrm{ZCIS} / \mathrm{ZnS}_{575}$ particles displayed a size of about $11 \pm$ $0.6 \mathrm{~nm}$. Hence, the blue-shift in the emission wavelength was not caused by smaller size and the shell growth induced a size enhancement, leading to a blue-shift instead of a red-shift.

Ionic liquid-soluble NCs via ligand exchange. Ligand exchange endows NCs with wide application potential, such as co-solvation with other polymers or provides covalent bonding for reaction with polymers. Herein, we could successfully cause thiol-ligand exchange by 3-mercaptopropyl-trimethoxysilane with the help of the ionic liquid 1-hexyl-3-methylimidazolium chloride as the solvent. It should be mentioned that we tried other ionic liquids, such as 1-ethyl-3-methylimidazolium chloride and 1-butyl-3-methylimidazolium chloride, but only the long chain 1-hexyl-3-methylimidazolium chloride could successfully aid in the ligand exchange from DDT to 3mercaptopropyl-trimethoxysilane for the ZCIS/ZnS nanoparticles and transfer the groups from toluene to the ionic
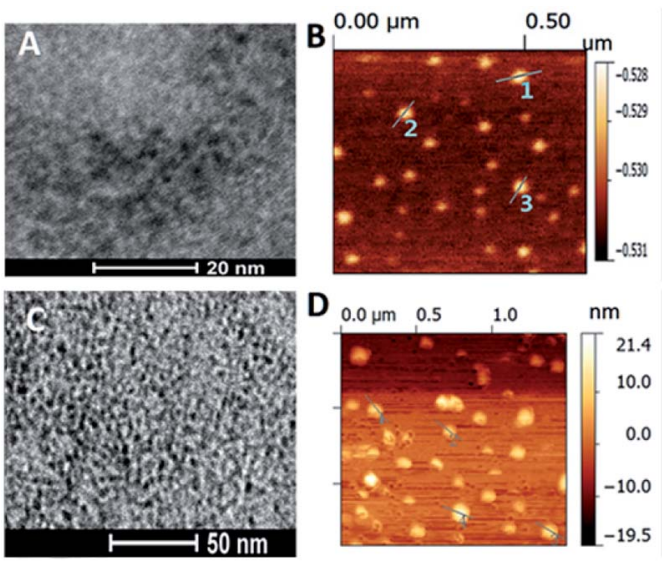

Fig. 6 Wide-field TEM and AFM images of $\mathrm{ZCIS}_{620}(\mathrm{~A}$ and $\mathrm{B}$ ). $\mathrm{Zn} / \mathrm{Cu} / \mathrm{In}$ $=0.2: 1: 1 \mathrm{in} 1 \mathrm{~h}$ at $230^{\circ} \mathrm{C}$ and its corresponding ZCIS/ZnS ${ }_{575}(\mathrm{C}$ and D) after 3 batches of $\mathrm{Zn}$ shell growth on the core. 


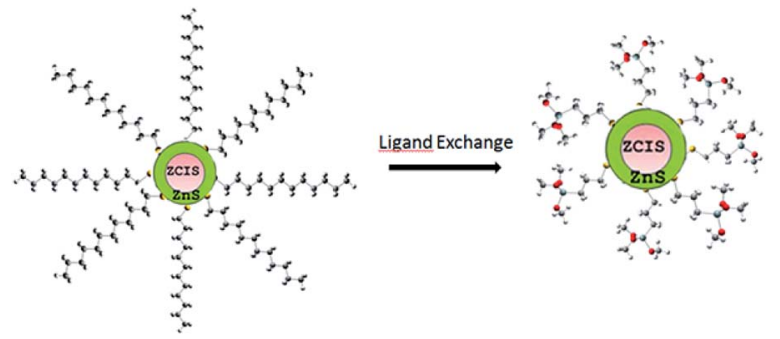

Fig. 7 ZCIS/ZnS capped with 1-dodecanethiol and ligand exchange with (3-mercaptopropyl)trimethoxysilane.

liquid 1-hexyl-3-methylimidazolium chloride. This solved the traditional problem of difficult DDT ligand exchange. The strong interactions between the mercapto group of MPTMS was the key factor in the successful ligand exchange of 1-dodecanethiol with MPTMS, as illustrated in Fig. 7. These nanoparticles can be converted from hydrophobic to hydrophilic via ligand exchange, thus rendering them readily dispersible in ionic liquids, as shown in Fig. 8. The ionic liquid-soluble ZCIS/ ZnS NCs could retain up to $70 \%$ of the luminescent intensity of the original oleophilic NCs (Fig. 8A). Moreover, the fluorescent emission peak with a narrow fluorescent region red-shifts by about $10 \mathrm{~nm}$, indicating that the red-shift was induced not by aggregation but rather by the changing environment. Fig. 8B shows luminescence in both toluene and ionic liquids under a UV lamp. The high luminescence of ZCIS/ZnS in the ionic liquid could be retained for several months without any observable quenching. This high PL stability and compatibility of the ionic liquid-soluble QDs is of special interest in the preparation of composite materials with polymers.

The ligand exchange behavior was further analyzed via GCMS. The standard calibration curve of 1-dodecanethiol was obtained using Br-decane as the internal standard. The equation $y=0.4929 x$ describes the ligand exchange behavior. Herein, different amounts of QDs were used for ligand exchange and the corresponding ligand 1-dodecanethiol remained in

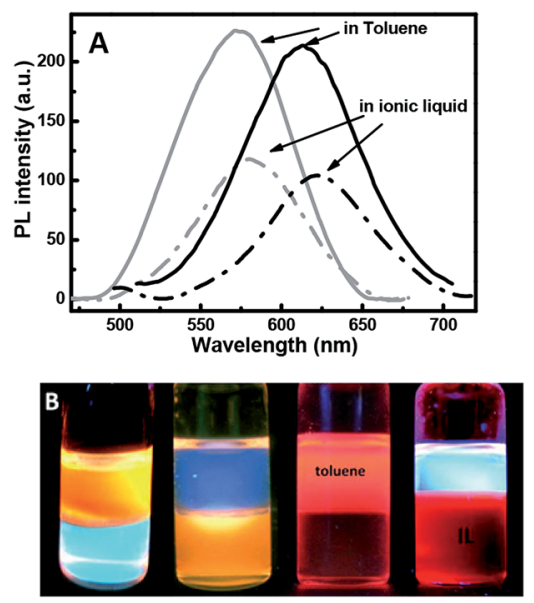

Fig. 8 Fluorescent spectra of ZCIS/ZnS core-shell samples before and after ligand exchange that stayed in toluene and ionic liquid $(A)$ and the corresponding luminescent photographs under a UV lamp (B).
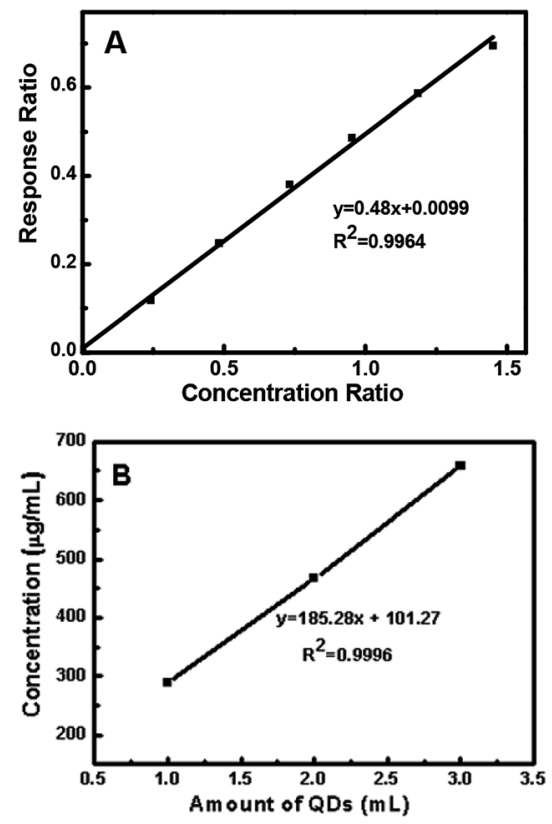

Fig. 9 GC-MS standard curve for 1-dodecanethiol (A) and the relationship between the QD concentration and responses for 1-dodecanethiol (B).

toluene after ligand exchange. This was found to follow the trend, as shown in Fig. 9. The remaining 1-dodecanethiol in the three samples showed a linear increase with increasing amounts of 1-mercapto- $n$-dodecane-capped QDs. According to the equation $y=185.28 x+101$ (ref. 49) $(y$ is the estimated concentration of the remaining 1-dodecanethiol in toluene $(\mu \mathrm{g}$ $\left.\mathrm{mL}^{-1}\right)$ ), 1-dodecanethiol was successfully exchanged by (3mercaptopropyl)trimethoxysilane. In addition, the linear trend implies that the exchange percentage of 1-dodecanethiol was the same every time.

\section{Conclusions}

ZCIS-alloyed NCs were prepared via a sulfur injection-free approach in the non-coordinating solvent octadecene in the presence of 1-dodecanethiol using copper iodide and indium and zinc acetates as the precursors. The band gap of the ZCISalloyed NCs can be conveniently tuned by adjusting the experimental variables, such as the $\mathrm{Zn}$ content, $\mathrm{Cu}$ : In ratio, dodecanethiol concentration, and reaction temperature. With the deposition of ZnS shells around the ZCIS cores, the PL QY increased substantially to a maximum value of $35 \%$, while the emission wavelength underwent a blue-shift and could be tuned from $518 \mathrm{~nm}$ to $810 \mathrm{~nm}$. The ionic liquid-soluble ZCIS/ZnS NCs obtained through ligand exchange could preserve the high PL emission efficiency, which was confirmed by GC-MS and PL measurements. Thus these novel, toxic-free fluorescent nanoparticles are promising in composite applications.

\section{Conflicts of interest}

There are no conflicts to declare. 


\section{Acknowledgements}

The authors would like to thank the internal cooperation from the University of Natural Resources and Life Sciences Vienna: Jose Toca-Herrera, David Schuster, Jaqueline Friedmann, Haizheng Zhong (School of Materials Science \& Engineering, Beijing Institute of Technology). The financial supports from the National Natural Science Foundation of China (51603059), Fundamental Research Fund for the Central Universities of China (106-4115100027), China Postdoctoral Foundation, and AnHui Provence Postdoctoral Foundation are gratefully acknowledged. The Christian Doppler Laboratory "Advanced cellulose chemistry and analytics" is thankfully acknowledged.

\section{References}

1 H. Abdullah and D. H. Kuo, ACS Appl. Mater. Interfaces, 2015, 7, 26941-26951.

2 B. Clemens, X. Chen, R. Narayanan and M. El-Sayed, Chem. Rev., 2005, 105, 1025-1029.

3 P. Alivisatos, Nat. Biotechnol., 2004, 22, 47-52.

4 B. K. Chen, H. Z. Zhong, W. Q. Zhang, et al., Adv. Funct. Mater., 2012, 22, 2081-2088.

5 T. Tomai, Y. Yasui and S. Watanabe, J. Supercrit. Fluids, 2016, 120, 448-452.

6 E. Belas, Uxa and R. Grill, J. Appl. Phys., 2014, 116, 103521.

7 Y. Chen, X. Guo and C. Xie, Int. J. Hydrogen Energy, 2018, 43, 13911-13920.

8 D. Pan, D. Weng, X. Wang, Q. Xiao, W. Chen, C. Xu, et al., Chem. Commun., 2009, 28, 4221-4223.

9 M. Kruszynska, H. Borchert, J. Parisi, et al., J. Am. Chem. Soc., 2010, 132, 15976-15986.

10 X. Tang, W. Cheng, E. S. G. Choo, et al., Chem. Commun., 2011, 47, 5217-5219.

11 X. Wang, D. Pan, D. Weng, C.-Y. Low, L. Rice, J. Han and Y. Lu, J. Phys. Chem. C, 2010, 114, 13406-13413.

12 W.-S. Song, J.-H. Kim, J.-H. Lee, H.-S. Lee, Y. R. Do and H. Yang, J. Mater. Chem., 2012, 22, 21901-21908.

13 Y.-H. Wang, X. Zhang, N. Bao, B. Lin and A. Gupta, J. Am. Chem. Soc., 2011, 133, 11072-11075.

14 Q. Liu, Z. Zhao, Y. Lin, P. Guo, S. Li, D. Pan and X. Ji, Chem. Commun., 2011, 47, 964-966.

15 W. Zhang and X. Zhong, Inorg. Chem., 2011, 50, 4065-4072.

16 J. Feng, M. Sun, F. Yang and X. Yang, Chem. Commun., 2011, 47, 6422-6424.

17 X. Wang, D. Pan, D. Weng, C.-Y. Low, L. Rice, J. Han and Y. Lu, J. Phys. Chem. C, 2010, 114, 13406-13413.

18 H. J. Pan, C. W. Lai and S. W. Chou, Mater. Express, 2012, 2, 224-232.

19 D. Pan, D. Weng, X. Wang, Q. Xiao, W. Chen, C. Xu, Z. Yang and Y. Lu, Chem. Commun., 2009, 40, 4221-4223.

20 H. Nakamura, W. Kato, M. Uehara, K. Nose, T. Omata, S. Otsuka-Yao-Matsuo, M. Miyazaki and H. Maeda, Chem. Mater., 2006, 18, 3330-3335.

21 L. De Trizio, M. Prato, A. Genovese, A. Casu, M. Povia, R. Simonutti, M. J. P. Alcocer, C. D. Andrea, F. Tassone and L. Manna, Chem. Mater., 2012, 24, 2400-2406.
22 E. J. Tyrrell and S. Tomic, J. Phys. Chem. C, 2015, 119, 1272021273.

23 N. Radychev, D. Scheunemann, M. Kruszynska, K. Frevert, R. Miranti, et al., Org. Electron., 2012, 13, 3154-3164.

24 Z. Bai, W. Ji, D. Han, et al., Chem. Mater., 2016, 28, 10851091.

25 M. Booth, A. Brown, S. Evans and K. Critchley, Chem. Mater., 2012, 24, 2064-2070.

26 W. Guo, N. Chen, Y. Tu, C. Dong, B. Zhang, C. Hu and J. Chang, Theranostics, 2013, 3, 99-108.

27 L. Li, T. J. T. Daou, I. Texier, T. K. Chi, T. T. Kim Chi, N. Q. Liem and P. Reiss, Chem. Mater., 2009, 21, 2422-2429.

28 K. Yu, P. Ng, J. Ouyang, M. Badruz Zaman, A. Abulrob, T. N. Baral, D. Fetehi, Z. J. Jakubek, D. Kingston, X. Wu, X. Liu, C. Hebert, D. M. Leek and D. M. Whitfield, $A C S$ Appl. Mater. Interfaces, 2013, 5, 2870-2880.

29 J. Feng, M. Sun, F. Yang and X. Yang, Chem. Commun., 2011, 47, 6422-6424.

30 C. C. Chang, P. H. Chen and C. M. Chang, J. Sol-Gel Sci. Technol., 2008, 47, 268-273.

31 S. S. Kim, J. E. Park and J. Lee, J. Appl. Polym. Sci., 2011, 119, 2261-2267.

32 A. S. Amarasekara and D. G. Reyes, Renewable Energy, 2019, 136, 352-357.

33 J. Wu, J. Zhang, H. Zhang, J. He, Q. Ren and M. Guo, Biomacromolecules, 2004, 5, 266-268.

34 R. P. Swatloski, S. K. Spear, J. D. Holbery, et al., J. Am. Chem. Soc., 2002, 124, 4974-4975.

35 G. Fan, C. Liao, T. Fang, M. Wang and G. Song, Fuel Process. Technol., 2013, 116, 142-148.

36 J. Hulsbosch, D. E. D. Vos, K. Binnemans, et al., RSC Adv., 2016, 6, 4053-4062.

37 M. M. Jaworska and A. Gorak, Mater. Lett., 2016, 164, 341343.

38 I. Bushra, M. Nawshad, R. Abdur, et al., Int. J. Polym. Mater. Polym. Biomater., 2019, 68, 590-596.

39 J. Li, B. Kempken, V. Dzhagan, et al., CrystEngComm, 2015, 17, 5634-5643.

40 D.-E. Nam, W.-S. Song and H. Yang, J. Colloid Interface Sci., 2011, 361, 491-496.

41 H. Zhong, Y. Zhou, M. Ye, Y. He, J. Ye, C. He, C. Yang and Y. Li, Chem. Mater., 2008, 20, 6434-6443.

42 S. L. Castro, S. G. Bailey, R. P. Raffaelle, K. K. Banger and A. F. Hepp, J. Phys. Chem. B, 2004, 108, 12429-12435.

43 M. Booth, A. Brown, S. Evans and K. Critchley, Chem. Mater., 2012, 24, 2064-2070.

44 J. Park and S.-W. Kim, J. Mater. Chem., 2011, 21, 3745-3750. 45 L. Li, T. J. Daou, I. Texier, T. K. K. Chi, N. Q. Liem and P. Reiss, Chem. Mater., 2009, 21, 2422-2429.

46 T. Pons, E. Pic, N. Lequeux, E. Cassette, L. Bezdetnaya, F. Guillemin, F. Marchal and B. Dubertret, ACS Nano, 2010, 4, 2531-2538.

47 L. Li, A. Pandey, D. J. Werder, B. P. Khanal, et al., J. Am. Chem. Soc., 2011, 133, 1176-1179.

48 J. Park and S.-W. Kim, J. Mater. Chem., 2011, 21, 3745-3750. 49 H. Y. Park, I. Ryu and J. Kim, J. Phys. Chem. C, 2014, 118, 17374-17382. 